\title{
Three-Year Follow-Up with the Bone Conduction Implant
}

\author{
Ann-Charlotte Persson $^{a, c}$ Sabine Reinfeldt ${ }^{b}$ Bo Håkansson ${ }^{b} \quad$ Cristina Rigato $^{b}$ \\ Karl-Johan Fredén Jansson ${ }^{b}$ Måns Eeg-Olofsson ${ }^{c}$ \\ ${ }^{a}$ Region Västra Götaland, Habilitation \& Health, Hearing Organization, Gothenburg, Sweden; ${ }^{b}$ Department \\ of Electrical Engineering, Chalmers University of Technology, Gothenburg, Sweden; ' Department of \\ Otorhinolaryngology Head and Neck Surgery, Institute of Clinical Sciences, Sahlgrenska Academy, University of \\ Gothenburg, Gothenburg, Sweden
}

\section{Keywords}

Hearing aids · Questionnaires · Bone conduction .

Implanted transducer - Transcutaneous device

\begin{abstract}
Background: The bone conduction implant $(\mathrm{BCl})$ is an active transcutaneous bone conduction device where the transducer has direct contact to the bone, and the skin is intact. Sixteen patients have been implanted with the $\mathrm{BCl}$ with a planned follow-up of 5 years. This study reports on hearing, quality of life, and objective measures up to 36 months of follow-up in 10 patients. Method: Repeated measures were performed at fitting and after 1, 3, 6, 12, and 36 months including sound field warble tone thresholds, speech recognition thresholds in quiet, speech recognition score in noise, and speech-to-noise thresholds for $50 \%$ correct words with adaptive noise. Three quality of life questionnaires were used to capture the benefit from the intervention, appreciation from different listening situations, and the ability to interact with other people when using the $\mathrm{BCl}$. The results were compared to the unaided situation and a Ponto Pro Power on a soft band. The implant functionality was measured by nasal sound pressure, and the retention force from the audio processor against the skin was measured using a
\end{abstract}

(C) 2020 The Author(s) Published by S. Karger AG, Basel

Karger Open access

This is an Open Access article licensed under the Creative Commons Attribution-NonCommercial-4.0 International License (CC BY-NC) (http://www.karger.com/Services/OpenAccessLicense), applicable to the online version of the article only. Usage and distribution for commercial purposes requires written permission. specially designed audio processor and a force gauge. $\boldsymbol{R e}$ sults: Audiometry and quality of life questionnaires using the $\mathrm{BCl}$ or the Ponto Pro Power on a soft band were significantly improved compared to the unaided situation and the results were statistically supported. There was generally no significant difference between the two devices. The nasal sound pressure remained stable over the study period and the force on the skin from the audio processor was $0.71 \pm$ $0.22 \mathrm{~N}$ (mean $\pm 1 \mathrm{SD}$ ). Conclusion: The $\mathrm{BCl}$ improves the hearing ability for tones and speech perception in quiet and in noise for the indicated patients. The results are stable over a 3-year period, and the patients subjectively report a beneficial experience from using the $\mathrm{BCl}$. The transducer performance and contact to the bone is unchanged over time, and the skin area under the audio processor remains without complications during the 3-year follow-up.

(C) 2020 The Author(s)

Published by S. Karger AG, Basel

\section{Background}

Implantable bone conduction devices (BCDs) are gaining increasing interest as a rehabilitative option for patients with conductive or mixed hearing loss and for patients with 


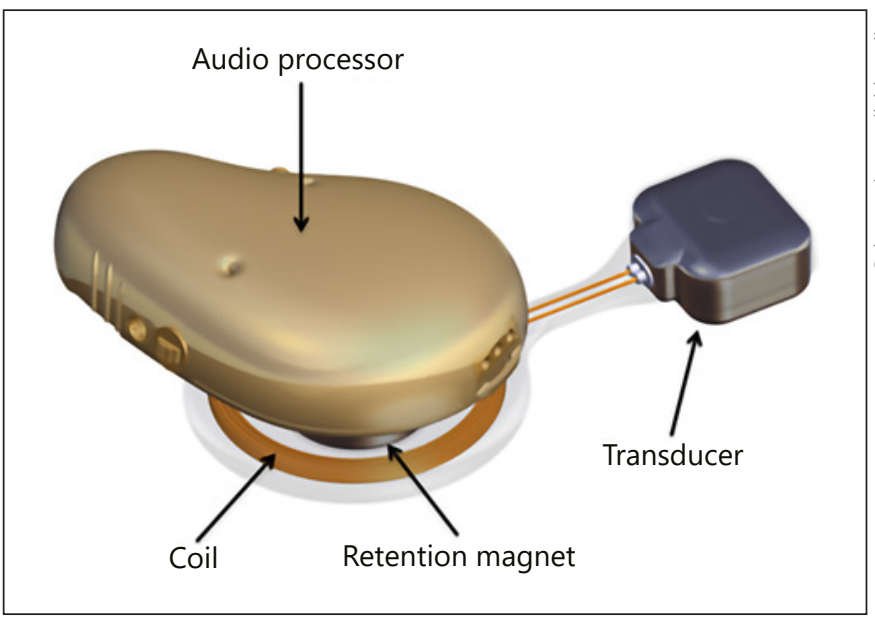

Fig. 1. Complete system of the bone conduction implant (BCI) showing the implanted part and the audio processor.

single-sided deafness. For almost 40 years, the percutaneous bone-anchored hearing aid has been a common choice of rehabilitation for these patient groups. In the percutaneous solution, the skin is penetrated by a titanium abutment (Ponto, Oticon Medical, Askim, Sweden, or Baha, Cochlear BAS, Mölnlycke, Sweden). Complications, such as skin inflammation or loss of the titanium screw implant, have driven the development towards implantable BCDs with an intact skin solution. The obvious advantage with an intact skin solution compared to the percutaneous solution is the possible decrease in skin complications. The major disadvantage is that implantable devices with an intact skin solution require a more extensive surgical procedure. Also, it becomes more complicated to perform magnetic resonance imaging (MRI) due to magnetically induced forces, and that the images of the brain will be distorted in regions close to the implant [Jansson et al., 2015].

Today, the implantable BCDs are divided into two main groups: direct drive systems, where the transducer has direct contact to the bone, and skin drive systems, where the transducer is attached onto the skin [Reinfeldt et al., 2015a]. Direct drive systems are also divided into percutaneous and active transcutaneous systems, where the active transcutaneous systems imply that the transducer is implanted under intact skin.

The bone conduction implant (BCI) is an active transcutaneous $\mathrm{BCD}$ and consists of an implanted part including the receiver coil, the retention magnet and the transducer, and an audio processor, which is retained on the skin by magnetic force [Hakansson et al., 2010; Taghavi et al., 2015] (Fig. 1).
Since 2012, 16 patients have been implanted with the BCI and enrolled in a clinical study with a protocol follow-up time of 5 years. Report of the first patient who received a BCI, and reports of the first 6 patients in a 6-month follow-up, have previously been published [EegOlofsson et al., 2014; Reinfeldt et al., 2015b]. In summary, the audiological outcomes in terms of tone audiometry and speech audiometry in quiet and in noise were significantly improved compared to the unaided situation and similar or better compared to a Ponto Pro Power (Oticon Medical, Askim, Sweden) on a soft band. Quality of life questionnaires also showed similar or improved results compared to the soft band device. The BCI surgery was regarded as being easy by the surgeon performing all surgical procedures in the current study. No serious adverse events occurred. The BCI has furthermore been tested in a 1.5-tesla MRI resulting in a $1-\mathrm{dB}$ deterioration of the maximum power output and 5\% loss of magnetic force of the retention magnet [Jansson et al., 2015]. On the MRI image, half of the brain was heavily distorted, but the distortion was expected and in the same range as for the Bonebridge (Med El, Innsbruck, Austria) and the Sophono (Medtronic, Dublin, Ireland). To test the long-term functionality, a test BCI has been exposed to continuous sounds $24 \mathrm{~h}$ a day, showing no signs of performance deterioration up to an estimated user time of 26 years $(12 \mathrm{~h}$ per day) [Fredén Jansson et al., 2019].

\section{Objectives}

The aim of this study is to report on audiometric results and patient-reported outcome measures from quality of life questionnaires for the first 10 patients at the 3-year follow-up. Furthermore, an analysis of the change in rehabilitation effect over time for these 10 patients is reported.

\section{Materials and Methods}

\section{Subjects}

Sixteen subjects have been included in the clinical trial of the BCI, 7 males and 9 females. In this study, results for the first 10 subjects, 5 males and 5 females, are given from their first 3 years with the BCI. Type of hearing loss, gender, implant side, and age at implantation of all subjects are shown in Table 1 . The inclusion criteria for the study were: (1) unilateral or bilateral conductive hearing loss with an air-bone gap of at least $20 \mathrm{~dB}$ (average over $500,1,000,2,000$, and $4,000 \mathrm{~Hz}$ ); (2) pure tone average bone conduction $\left(\mathrm{PTA}_{4, \mathrm{BC}}\right)$ of $30 \mathrm{~dB}$ HL (average over 500, 1,000, 2,000 and $4,000 \mathrm{~Hz}$ ) or better; (3) To either reject or be unable to use a conventional air conduction hearing aid; (4) to be accessible for multiple follow-up visits according to the protocol and be motivated to be one of the first subjects using the BCI. 
Table 1. Type of hearing loss, gender, implant side, and age at implantation described for each patient

\begin{tabular}{rllll}
\hline Patient & Hearing loss & Gender & Implant side & $\begin{array}{l}\text { Age at implantation } \\
\text { of BCI, years }\end{array}$ \\
\hline 1 & unilateral mixed loss & F & R & 42 \\
2 & bilateral conduction loss & M & L & 48 \\
3 & unilateral conduction loss & M & R & 18 \\
4 & bilateral conduction loss & F & L & 67 \\
5 & bilateral mixed loss & F & R & 48 \\
6 & bilateral conduction loss & M & L & 49 \\
7 & unilateral conduction loss & M & L & 20 \\
8 & bilateral conduction loss & M & L & 49 \\
9 & unilateral conduction loss & F & L & 20 \\
10 & bilateral conduction/mixed loss & F & L & 21 \\
\hline
\end{tabular}

\section{Overall Procedure}

After inclusion of a patient, a date for BCI surgery was decided. Before the BCI surgery, a reference device on a soft band was fitted and used for 4 weeks followed by audiometric tests and quality of life questionnaires. The same tests and questionnaires were later used for the implanted BCI.

The BCI was implanted under general anesthesia. A detailed description of the surgical procedure can be found in previously published studies [Eeg-Olofsson et al., 2013; Reinfeldt et al., 2015b].

One month after surgery, fitting of the BCI audio processor took place. On this occasion and at all follow-up visits, audiometric tests and objective measures were performed. The follow-up visits took place $1,3,6,12$, and 36 months after fitting. The audiometric test order was randomized at all follow-up visits for each patient to minimize the order effect; also the order of the blocks (unaided-aided) was varied. The different speech lists for each speech test were randomized to be evenly spread at both the unaided and the aided test situation.

The Abbreviated Profile of Hearing Aid Benefit (APHAB) and the Glasgow Benefit Inventory (GBI) questionnaires were completed by the patients after using the reference device for 1 month, and 6,12 , and 36 months after the BCI fitting. At the 36 months visits, the International Outcome Inventory of Hearing Aids (IOIHA) was added.

\section{Reference Device}

A reference device, Ponto Pro Power (Ponto, Oticon Medical, Askim, Sweden) on a soft band, hereafter referred to as the reference device, was fitted using in-situ thresholds, including skin compensation of $10-\mathrm{dB}$ higher gain in the high frequency range. The reference device was individually fitted for each patient with one general program, the algorithm NAL-NL1 in the software Genie Medical (Oticon Medical, Askim, Sweden), using omnidirectional microphones, and all automatic functions, such as feedback manager and noise manager, turned off. The generic resonance frequency at approximately $750 \mathrm{~Hz}$ that appears in the direct drive percutaneous application is electronically damped in the Ponto device, and it was not possible to turn this dampening effect off. The reference device was used for 4 weeks and evaluated with audiometric tests and two questionnaires, APHAB and GBI.

Three-Year Follow-Up with BCI

\section{Fitting of the BCI}

For fitting of the audio processor, a computer-based software (On Semiconductor, Phoenix, AZ, USA) was used. No specific fitting algorithms taking the subjects' hearing thresholds into account have been developed so far. Individual adjustments were made at fitting, and if necessary, more adjustments were made at follow-up visits. The BCI audio processor has six pre-set program options, but after some time, it turned out that mainly the first program (Pr 1) was used, and that the second program $(\operatorname{Pr} 2)$ was used occasionally in noisy environments. In Pr 1, the most preferred settings regarding frequency shaping and volume control were implemented. Those settings were determined during the initial fitting of the device generally based on an assumed target giving maximum linear gain over all frequencies while still allowing a reasonably large headroom before saturation at $70 \mathrm{~dB}$ SPL input sound level. This headroom was set to be larger at lower frequencies (approx. $20 \mathrm{~dB}$ below 1,000 Hz) and smaller at higher frequencies (approx. $10 \mathrm{~dB}$ above $1,000 \mathrm{~Hz}$ ) in order not to distort speech level sounds. The patients' audiograms and their in-situ thresholds obtained with the reference device were also used for determining frequency-specific adjustments. Finally, the preferred volume control setting was decided and pre-set for start-up. In the initial fitting, as well as in later follow-up visits, adjustments were made based on an interaction with the patients and their experience. In some patients, compression was later introduced to reduce the negative influence of hearing their own voice. In $\operatorname{Pr} 2$, all settings were based on $\operatorname{Pr} 1$, but with a general reduction of the high frequency gain in order to offer a more comfortable sound in noisy environments. For all audiometric tests in this clinical study, $\operatorname{Pr} 1$ and the preferred volume control setting were used.

\section{Audiometric Tests}

The audiometry was done in a sound insulated test room of 16 $\mathrm{m}^{3}$, and the equipment was calibrated according to standard procedures. All audiometric tests were performed in a sound field, where all signals (tones, speech and noise) were played from a loudspeaker $1 \mathrm{~m}$ in front of the patient. The methods used were (a) warble tone thresholds, (b) speech recognition score (SRS) in noise with phonemically balanced word lists at $4 \mathrm{~dB}$ higher level than the noise, measured at $63 \mathrm{~dB}$ SPL (ISO: 8253-3 [2012]), (c) speech recognition thresholds (SRT) in quiet using Swedish spondees according to ISO 8253-3 [2012], and (d) signal-to-noise ratio thresh- 
olds (SNR thresholds) with five-word Hagerman sentences at a fixed level of $63 \mathrm{~dB}$ SPL and adaptive noise level to reach $50 \%$ speech intelligibility [Hagerman and Kinnefors, 1995]. The speech and noise levels were monitored using an AC40 audiometer (Interacoustics AS, Assens, Denmark).

During all measurements, blocking of the non-test ear with an earplug (E-A-R Classic Soft) and an earmuff (Peltor, 3M Svenska $\mathrm{AB}$, Sollentuna, Sweden) was applied for all subjects with contralateral hearing thresholds better than or similar to the thresholds of the implanted ear. The purpose of blocking the non-test ear is to attenuate the air conducted sound, while letting the bone-conducted sound be perceived by both cochleae, like in real-life situations. The earplug was inserted deep in the ear canal to minimize the occlusion effect [Stenfelt and Reinfeldt, 2007].

\section{Patient-Related Outcome Measures}

The questionnaires used were APHAB, GBI, and IOI-HA.

APHAB focuses on the difference between unaided and aided condition and has four subscales: Ease of Communication (EC), Reverberation (RV), Background Noise (BN), and Aversiveness of sounds (AV) [Cox and Alexander, 1995]. The first three subscales $\mathrm{EC}, \mathrm{RV}$, and $\mathrm{BN}$ address speech understanding in various everyday environments. The fourth subscale, AV, quantifies negative reactions to environmental sounds. APHAB produces scores in percentage between 0 to $100 \%$ for both aided and unaided performance as well as hearing aid benefit. A lower score represents a more beneficial situation. However, the score will be presented as an improvement compared to the unaided condition. The unaided condition is asked for before surgery in comparison with the reference device, and after surgery at every follow-up visit in comparison with the BCI.

The GBI questionnaire measures patient benefit in general as a change in health status after an otorhinolaryngological surgical or medical intervention [Robinson et al., 1996]. GBI has three subdomains: general, social, and physical benefit. The responses are scaled and averaged to give a score between -100 and +100 , where everything above 0 , which represents the unaided condition, indicates a benefit from the intervention. The purpose of the GBI is to show if there is a general benefit or a change in health status after a surgical or medical intervention.

IOI-HA was used only at the 36-month follow-up visit. IOI$\mathrm{HA}$ is a seven-item questionnaire with issues that concern aspects of hearing as daily use of the hearing aid, benefit, satisfaction, participation difficulties, impact on others and quality of life. The main goal of the IOI-HA was to develop a short questionnaire suitable for translation into different languages and for comparison of the different results between countries [Cox and Alexander, 2002; Kramer et al., 2002]. The focus of IOI-HA is primarily on items associated with the wearer's experience of using the hearing aid separated from specific listening situations, and also limitations in daily activities. Thus, it should be used as a complement to other subjective measures [Cox and Alexander, 2002]. Cox and Alexander [2002] identified two factors where items "Use time," "Benefit," "Satisfaction," and "Quality of life" were clustered into Factor 1, while "Residual activity limitation," "Residual participation restriction," and "Impact on others" were clustered into Factor 2. Patients were also given an extra question concerning how many days a week they used their BCI. Low scores signalize not sufficient rehabilitation and high scores a successful rehabilitation, although there are no normative data to support either re- sults. For the item "Hours of daily use" the alternatives are: no use, less than $1,1-4,4-8 \mathrm{~h}$, and more than $8 \mathrm{~h}$, corresponding to a score from 1 to 5 .

\section{Objective Measures}

Audio Processor Force Attachment to the Skull

At every visit after implantation, the retention force to the implant magnet was measured to make sure that the force was not too high for the patient. A reference audio processor was used for these measurements, specially designed with the standard magnet strength and hooks to attach a force gauge model FG-5000A from Lutron Electronic Enterprise co., LTD., China. The retention force was defined as the peak force required to pull the audio processor perpendicular to the skull bone surface until it was released from the head. Above $0.7 \mathrm{~N}$, changing to a weaker magnet was considered. If any inconvenience or symptoms from a too high retention force were noted, i.e. pain, redness or inflammation of the skin area of the audio processor, the magnet was changed to a weaker strength.

\section{Nasal Sound Pressure}

In order to have functionality verification of the BCI implant at surgery, and also to have an objective measure on the implant transmission to bone over time, nasal sound pressure (NSP) was obtained. At surgery, before closing the wound, and at every follow-up visit, an omni-directional microphone (EM-23346, Knowles Electronics, Itasca, IL, USA) attached to a probe tube within an EAR Classic ear-plug (3M, Maplewood, MN, USA), was inserted in the ipsilateral nostril. An amplitude-modulated driver stage was developed to drive the inductive link using an Agilent 35670A (Keysight Technologies, Santa Rosa, CA, USA) as an FFT analyzer and speech frequency signal generator, and an Agilent 33220A (Keysight Technologies) as a carrier frequency and amplitude modulation signal generator. The driver stage was attached to the skin like the ordinary audio processor. With the BCI in place in direct contact to the bone, vibrations are transmitted throughout the skull and radiating into all cavities, including the nasal cavities, creating a sound pressure whose frequency response was measured using the Agilent 35670A in the frequency range of 0.1$10 \mathrm{kHz}$. Reinfeldt et al. [2019] provide a more detailed description of the NSP layout and calculations.

\section{Data Analysis}

The improvement given by the BCI and the reference device in warble tone thresholds was calculated as the difference between the measurements at the 3-year follow-up visit and the average unaided condition. The statistical significance of the improvement at each frequency was tested at significance level $\alpha<5 \%$ using the Wilcoxon signed rank test with the null hypothesis of no difference between the aided and the unaided conditions. No correction for multiple comparisons was deemed necessary since the tests are intended to identify at which frequencies the improvement differs from zero rather than to pairwise compare each frequency. The Wilcoxon signed rank test was used also to compare the two aided conditions, i.e. BCI compared to the reference device.

Improvements in SRS and SRT were analyzed analogously. SNR thresholds were considered only in the aided condition due to the fact that it was not possible to measure the unaided condition at the same speech level for all the patients.

Linear regression analysis was performed in order to study possible trends over time, and the regression lines coefficients $(\mathrm{k})$ were 


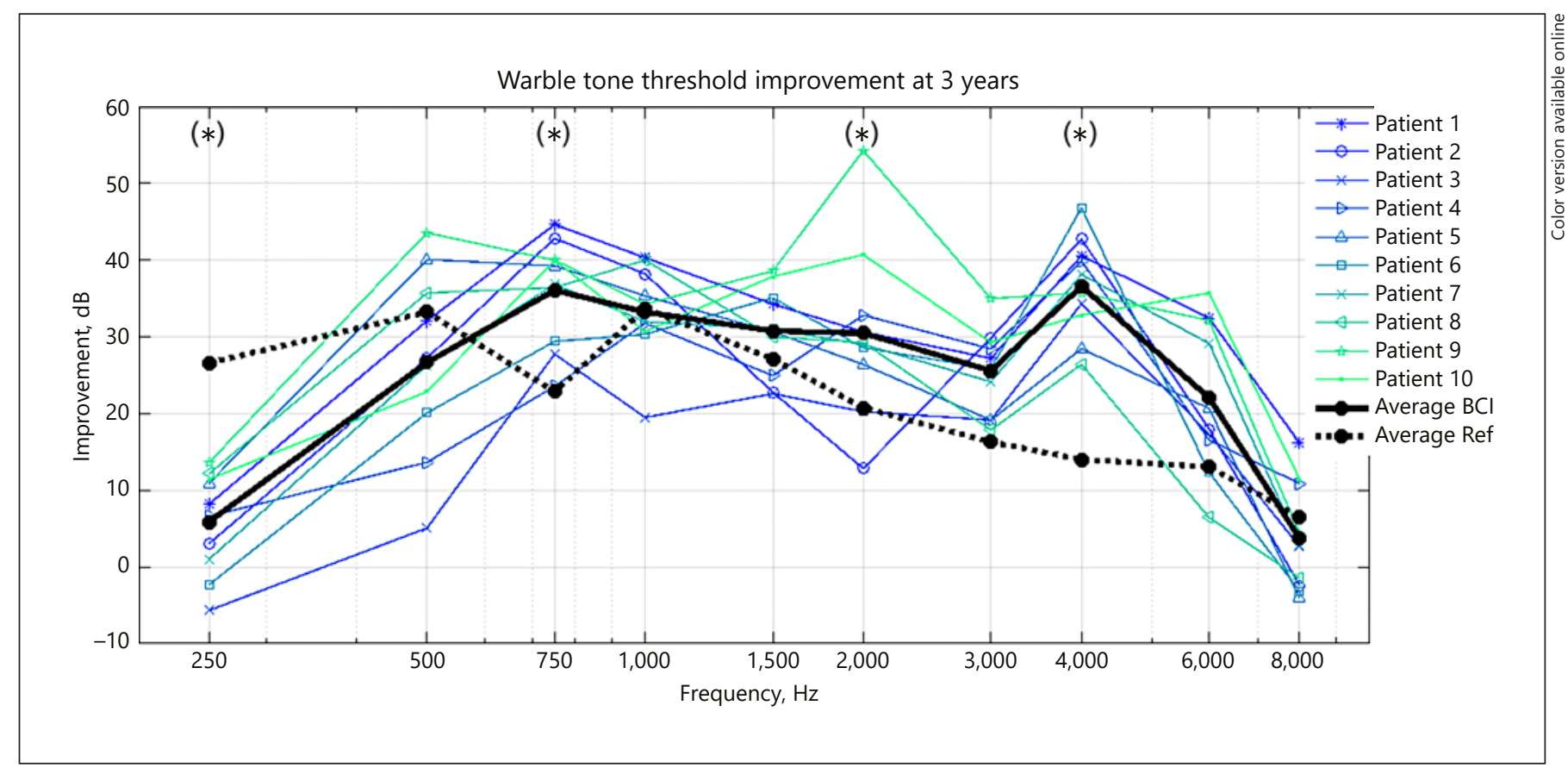

Fig. 2. Mean warble tone threshold improvement of all patients for the BCI together with their average (thick solid line) and the average for the reference device (thick dotted line) at 3-year follow-up. Statistically significant different results between the BCI and the reference device were found at 250, 750, 2,000, and 4,000 $\mathrm{Hz}$, marked with an asterisk.

used as an indication of increasing $(\mathrm{k}>0)$, decreasing $(\mathrm{k}<0)$ or stable results $(\mathrm{k} \sim 0)$. Linear models were fitted for each patient, and the obtained coefficients were tested with the Wilcoxon signed rank test to check for potential group trends. For 2 patients, data from one follow-up visit was missing (12 months for patient 3 and 3 months for patient 6); the regression coefficient in these cases was computed based on the remaining available data.

Data analysis was performed with MatLab R2018b (MathWorks Inc., Natick, MA, USA).

\section{Results}

\section{Audiometric Results}

Ten patients have completed the 3-year follow-up, and in Figure 2, it is shown that the warble tone thresholds at the 3-year follow-up for the $\mathrm{BCI}$ and the reference device provide an average $\mathrm{PTA}_{4}(500,1,000,2,000$, and 4,000 $\mathrm{Hz}$ ) improvement of $31.7 \mathrm{~dB}$ (median: $31.6 \mathrm{~dB}$, range: 19.8-42 dB) and $25.4 \mathrm{~dB}$ (median: $28.4 \mathrm{~dB}$, range: $8.1-$ $35.7 \mathrm{~dB}$ ), respectively, over the unaided condition. The Wilcoxon signed rank test shows that the improvement is statistically significant $(p<0.05)$ at all frequencies, except at 250 and $8,000 \mathrm{~Hz}$. The improvement calculation was based on the average unaided tone threshold of all test occasions. There was no change in statistical significance when including unaided results only at the 3-year follow-up. Comparing the BCI and the reference device, the BCI results seem generally better although supported by a statistically significant difference only at 750, 2,000, and $4,000 \mathrm{~Hz}$.

There is a statistically significant improvement in SRT and SRS over the unaided situation for the BCI and for the reference device at the 3-year follow-up, as shown in Figure 3. Compared to the unaided condition, the average improvement with the $\mathrm{BCI}$ was $27.5 \mathrm{~dB}$ in SRT (median: $26.6 \mathrm{~dB}$, range: $17.5-41 \mathrm{~dB}$ ) and $42.6 \%$ in SRS (median: $41 \%$, range: $28-58 \%$ ). With the reference device, the SRT average improvement was $27.2 \mathrm{~dB}$ (median: $28.6 \mathrm{~dB}$, range: $7.9-37.3 \mathrm{~dB}$ ), and the SRS was improved by $43.8 \%$ on average (median: $46 \%$, range: $20-62 \%$ ).

Nine out of 10 patients showed a better SNR threshold with the BCI compared to the reference device (Fig. 3, rightmost panel), but according to the Wilcoxon signed rank test, there was no statistically significant difference between the devices. 
Fig. 3. Aided SRT and SRS (left and middle boxplots) for the BCI (blue) and the reference device (pink) compared to the unaided condition. The boxplot shows the 25-75 percentiles where the horizontal line is the median. Mean is depicted as a green star and data points are grey dots. SNR thresholds (right) for each patient are shown as blue stars for the BCI and pink stars for the reference device. All results in Figure 3 have been obtained from the 36-month follow-up visit.
Fig. 4. The average APHAB score for the 10 patients using the reference device and the BCI.
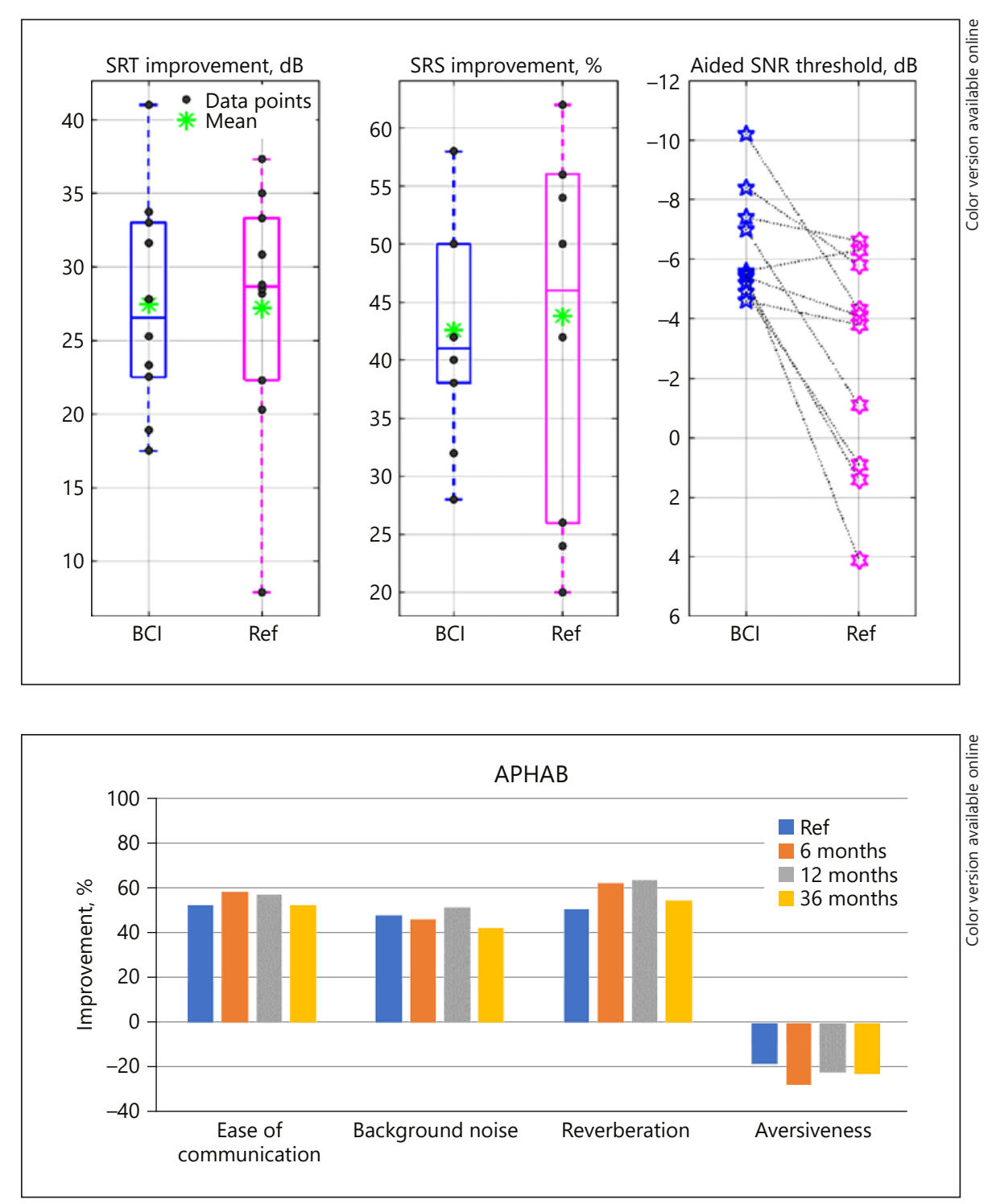

\section{Patient-Related Outcome Measures}

The results from APHAB (divided in four subscales: $\mathrm{EC}, \mathrm{BN}, \mathrm{RV}$, and $\mathrm{AV}$ ) are presented as improvement from the unaided to the aided condition over time in Figure 4. For the subscales EC, BN and RV, there is a statistically significant difference between unaided and both the reference device and the BCI for all follow-up visits, but for the subscale AV, the Wilcoxon signed rank test shows no statistically significant difference. There was no statistically significant difference between the reference device and the BCI.

Figure 5 presents results for the GBI over time. The GBI results show a statistically significant benefit for both devices except in physical health for the reference device.
There was no statistically significant change over the 3-year follow-up between the BCI and the reference device.

The questionnaire IOI-HA was used from the 36month follow-up (Fig. 6). The mean score for all questions was 4.2. In factor 1 (Daily use, Benefit, Satisfaction, and Quality of life), the patient score was between 3.9 and 4.6 , and in factor 2 (Residual activity limitation, Restriction in participation, and Impact on others), the patient score was between 3.7 and 4.1. The BCI was used 5.6 days a week, on average.

To visualize the distribution of responses, Figure 7 presents the percentage of patient responses to each question according to score. 
Fig. 5. GBI scores after using the reference device and after using the BCI for 6,12 , and 36 months. The unaided situation is set to 0 .

Fig. 6. IOI-HA results after using the BCI displayed as an average value for the 10 included patients. Use, Hours of daily use; Ben, Benefit; RAL, Residual activity limitation; Sat, Satisfaction; RPR, Residual participation restriction; Ioth, Impact on others; QoL, Quality of life.
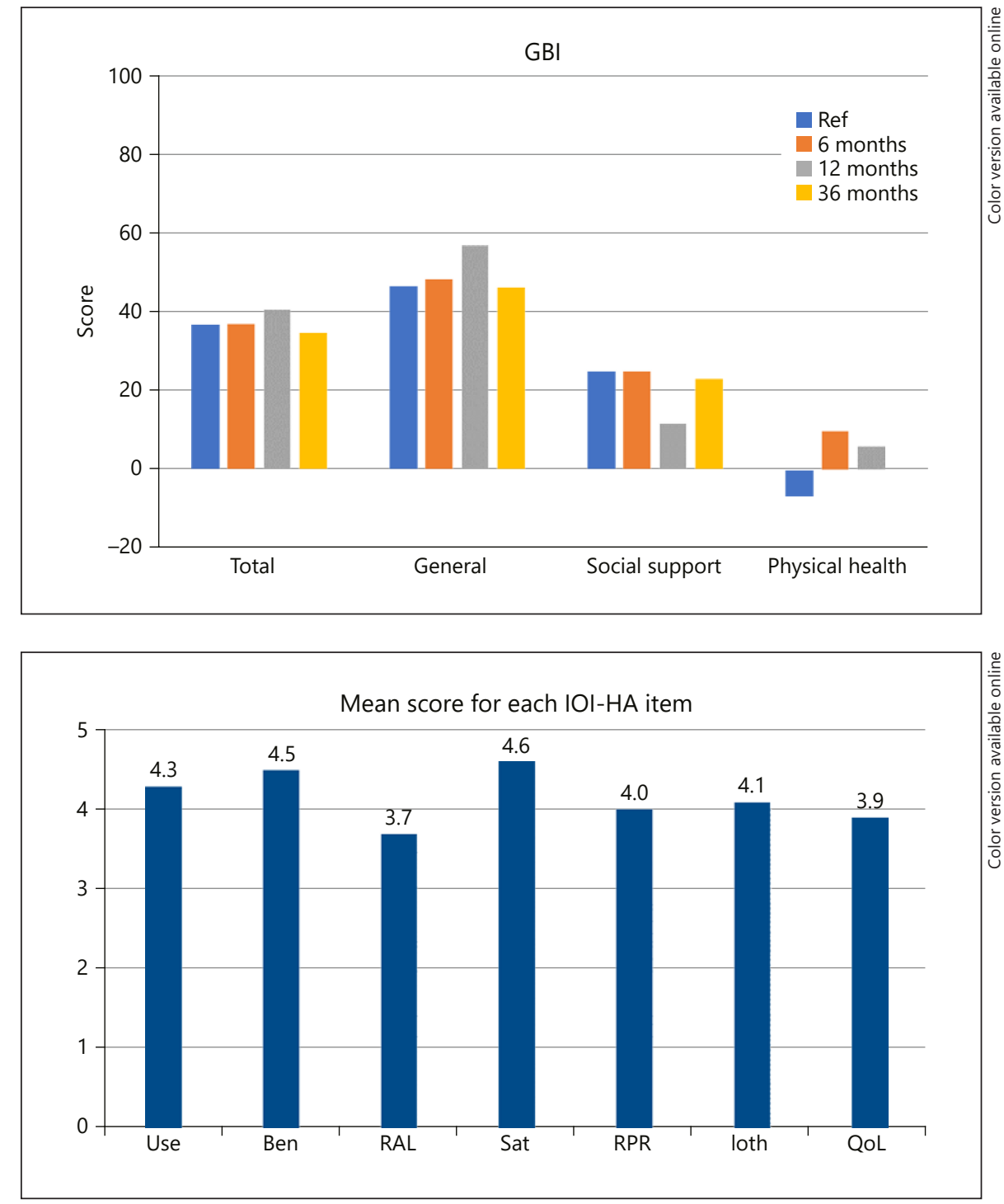

\section{Audio Processor Force Attachment to the Skull and NSP}

None of the patients had any skin problems related to the retention force, although patient 2 changed to a stronger retention magnet at the 3-month follow-up, but changed it back again to a weaker magnet at the 12- and 36-month follow-ups. Patient 4 changed to a weaker magnet strength at the 36-month follow-up. The retention force was found to increase during the first month of use in all patients, probably as a result of compression of the skin and soft tissues over the retention magnet. The average retention force during 3 years of use for all patients was $0.71 \pm 0.22 \mathrm{~N}$ (mean $\pm 1 \mathrm{SD}$ ). The average retention force increase during the first month was $0.14 \pm$ $0.11 \mathrm{~N}$.
The NSP obtained at surgery generally showed different results between the patients. Based on NSP in a pilot study on 20 healthy subjects [Shirinkar and Ghoncheh, 2013], the anticipated frequency response was used to decide whether the implant was considered to be functioning and if implant-to-bone contact was considered sufficient, which in this clinical study was the case for all patients at surgery before closing the wound and at all follow-up visits. The results at surgery varied from the follow-up measurements in all patients [Reinfeldt et al., 2019], as can be seen in Figure 8, where the NSP from patient 4 is shown as an example. In the follow-up NSP measurements, there were large interindividual differences, but low intraindividual variability. 


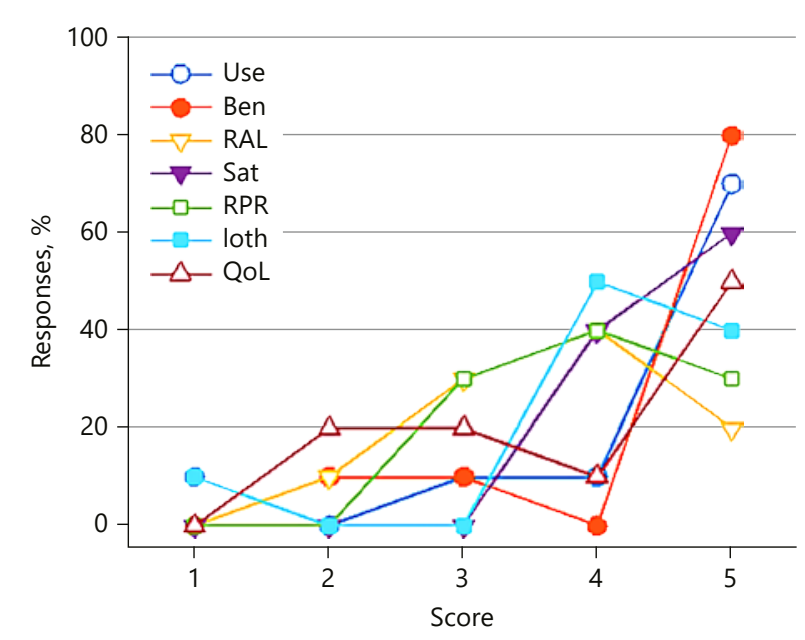

Fig. 7. Distribution of responses in percent for each IOI-HA item. Use, Hours of daily use; Ben, Benefit; RAL, Residual activity limitation; Sat, Satisfaction; RPR, Residual participation restriction; Ioth, Impact on others; QoL, Quality of life.

\section{Discussion}

For the 10 patients who have worn their BCI device for 3 years, there are no significant changes in unaided BC thresholds. On average, audiometric results show a statistically significant advantage with the BCI over the unaided situation, and similar or better results compared to the reference device. There are some differences over time in favor of the BCI compared to the reference device. The questionnaires indicate an overall satisfaction with the BCI compared to the unaided situation.

\section{Audiometric Results over Time}

An important purpose of the audiometric results is to clarify if there has been a change in improvement in the BCI compared to the unaided situation over time. In Figure 9 , sound field warble tone average improvement $(500$, $1,000,2,000$, and 4,000 Hz) is shown for 0-, 1-, 3-, 6-, 12-, and 36-month follow-ups. For each patient, a linear model is fitted, and the obtained slopes are tested with the Wilcoxon signed rank test to determine if the trend over time at one specific frequency is different from zero. No significant trend was detected in any of the four analyzed frequencies. In the same way, Figure 10 displays SRT, SRS, and SNR thresholds over time. The results are stable, and the slopes indicate small individual changes although not statistically significant at a group level.
The intrasubject variations in both tone and speech audiometry are generally quite small. The results are in every test occasion better for both the BCI and the reference device compared to the unaided situation. In a more detailed scrutiny of the difference between the BCI and the reference device, the latter not seldom leads to a better result, although the average and median results are in favor of the BCI, except for the SRS results. It is important to emphasize that the reference device results are only obtained at one occasion before the BCI surgery, and then not repeated. This methodology can imply a higher risk of outliers compared to performing repeated measurement during the study period. As always, when obtaining results from subjective measures, many factors can affect the results. Interestingly, the SRS improvement at the 3 years' visit for the $\mathrm{BCI}$ is worse compared to the improvement of the reference device. The corresponding result for SRT is highly similar, and for SNR thresholds, on the contrary, better. The advantage of using more than one speech test yields results that can imply a broader understanding of the concept of speech perception. There is no agreement on which of the tests is the most representative of the rehabilitation quality. Therefore, it is of major importance not to draw specific conclusions from rather limited differences in speech results when comparing devices within a study, or when comparing devices between different studies with similar test batteries. Authors and clinicians must acknowledge that these tests are still blunt, and results should be interpreted with care. For the current study, the audiometric results show a significant improvement for the BCI and the reference device compared to the unaided situation, while the comparison between the BCI and the reference device shows similar results.

\section{Quality of Life}

Although widely used in Swedish hearing aid research, $A P H A B$ and GBI are not validated for the Swedish language, which is suboptimal from a scientific point of view. IOI-HA is validated for the Swedish language. The questionnaires included in this study are constructed based on the aim of the questionnaire and what the constructors believe is important to highlight. It is important however to be critical about what is missing in the questionnaires. To be able to understand what the patient believes is the most important issue, a qualitative approach with interviews in focus groups or deep interviews with, in this case, patients who have undergone a surgical intervention for an implantable hearing aid, might be considered. The qualitative approach in constructing questionnaires of 
Fig. 8. Typical NSP (nasal sound pressure) results, in this case for patient 4 . The thick solid blue line represents the measurement at surgery, and the six thin dotted lines are measurements at follow-up visits, from fitting to 36 months. The average follow-up result is shown as a thick solid black line, and the average noise floor as a thick dotted black line.
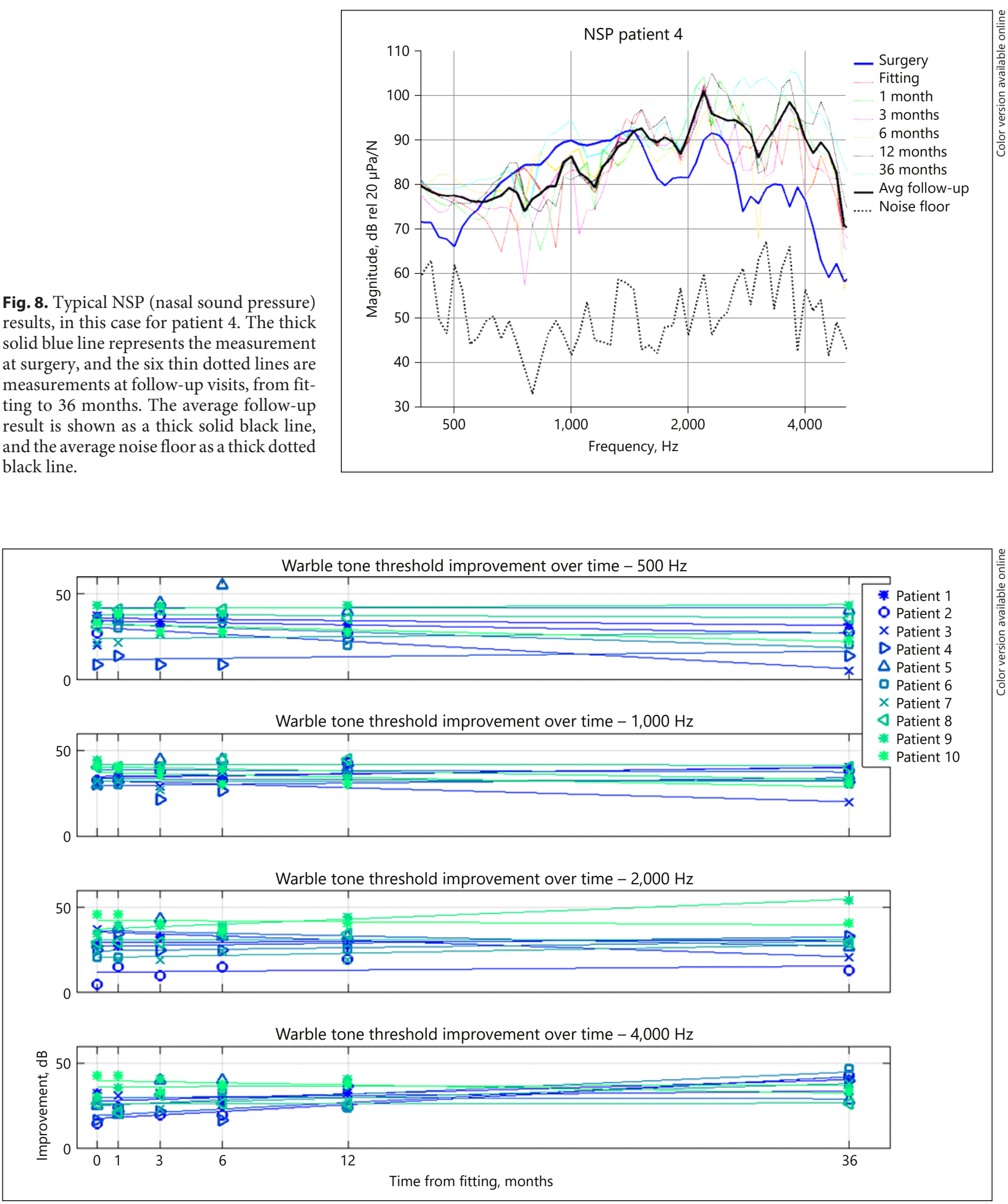

Fig. 9. Linear models are shown for 500, 1,000, 2,000, and 4,000 Hz, where each line represents the linear model for a single patient. 


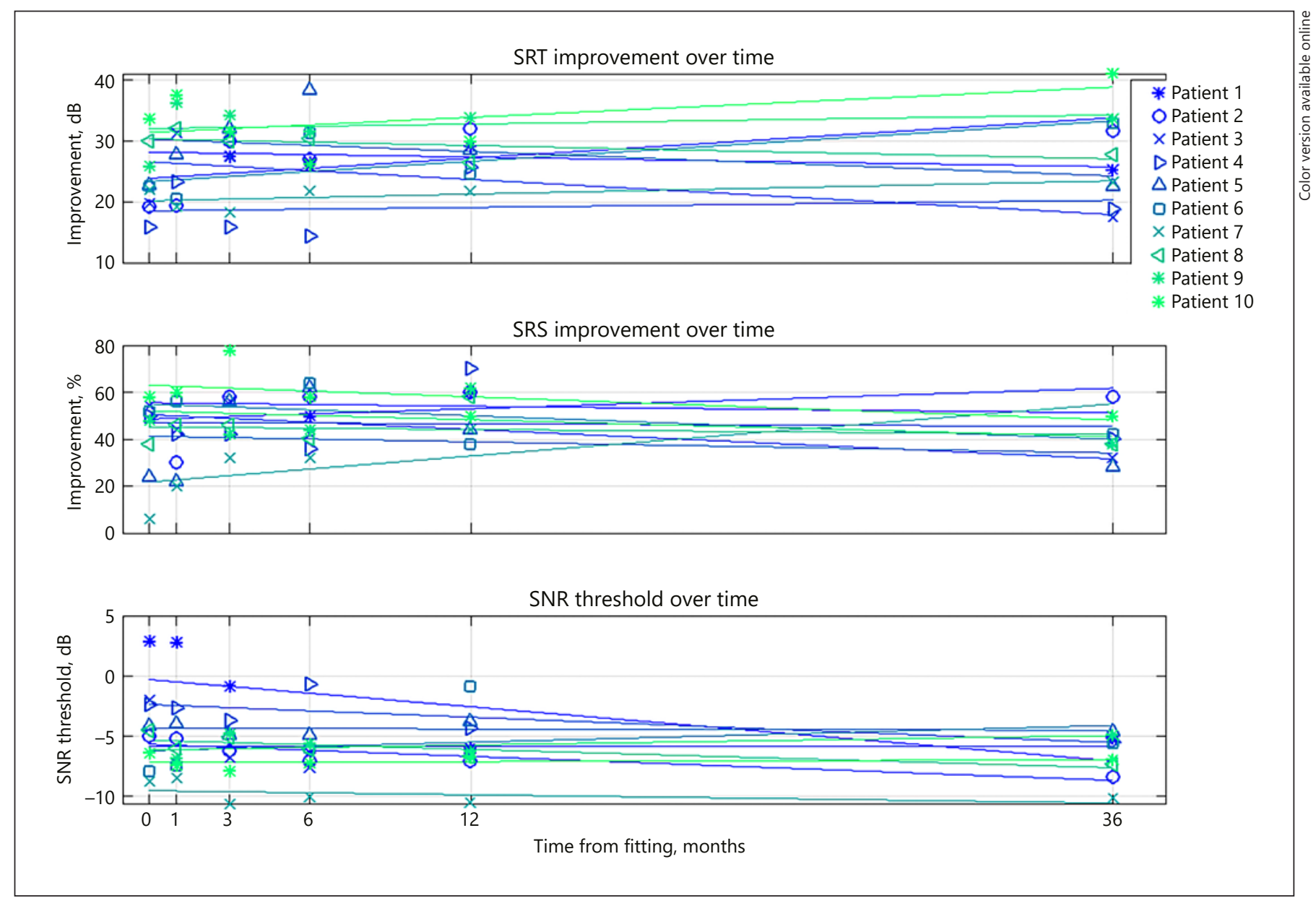

Fig. 10. A linear model is shown for SRT, SRS, and SNR (top, middle, and bottom) where each line represents a single patient.

different types is gaining both interest and popularity. All tests used in the current study have limitations, which accounts for tone- and speech audiometry, but also for the questionnaires. To be able to cover for the weaknesses, the authors have included multiple tests for both speech tests and questionnaires. Using this methodology, it seems reasonable that the conclusions are less affected by outliers due to the test itself.

Over time, there are only minor differences in the questionnaire scores. APHAB and GBI results show that both the BCI and the reference device score statistically significantly better compared to the unaided situation except for the AV domain in APHAB. Interestingly, in GBI, the reference device shows no significant difference compared to the unaided situation in the physical health domain. Although not statistically supported, there is a trend for improved physical benefit for the BCI compared to the reference device. The main purpose of developing active transcutaneous devices is that the transducer is attached directly to the bone with intact skin above. It is assumed that the active transcutaneous solution can provide both better comfort and less skin complications compared to percutaneous and skin drive passive transcutaneous solutions. The finding in the GBI physical health domain might support this assumption.

The IOI-HA was only used once in this study, at the 36-month follow-up. The partition of the results into factors can be helpful to identify the experience of wearing the BCI (factor 1), and the role the BCI plays when interacting with people around the user (factor 2). In this study, the scores are regarded as high, above 2 for both factors with slightly higher results for factor 1 [Cox and Alexander, 2002]. 
By using all three questionnaires, the authors believe that a general satisfaction was captured regarding listening situations, health status, and benefit for the patients alone and in interaction with other people.

\section{Audio Processor Attachment to Skin and Transducer Contact to Bone}

The NSP allows to verify the implant functionality before closing the wound and to follow sound transmission over time. There is always some uncertainty whether implantable electronics are functioning properly after implantation, and using a tool for verification can eliminate risks, time, and costs which will follow from an additional avoidable surgery. Another tool to control the implant functionality used in the current BCI study is a batteryoperated audio processor that produces a short melody of tones when the implant is functioning properly and has good contact to the bone. Schnabl et al. [2014] placed a microphone on the forehead in both cadavers and live humans and could in that way verify the implant to bone contact and the functionality of an implanted Bonebridge [Schnabl et al., 2014]. No matter which method is used, a verification tool is recommended when doing this kind of surgical and audiological intervention.

The NSP measurement was done in all patients at every follow-up visit and confirms that the bone to implant contact is intact, even if results from audiological testing in some patients vary more than expected. The NSP results have been consistent in all patients, and the measurement does not seem to be sensitive to, e.g., a swollen nasal mucosa, although no such testing has been a parameter in the NSP study [Reinfeldt et al., 2019]. The reasons for the different results at surgery and at the subsequent follow-ups are not known. The situation at surgery when a patient is anesthetized and has a tracheal tube in place probably comprises the explanation, but the mechanisms for the different results are still unknown.

The force of BCI audio processor retention over the implant was on average $0.71 \mathrm{~N}$, which is lower compared to a skin drive passive transcutaneous device (e.g., 1.2 N in Cochlear Baha Attract) [den Besten et al., 2019]. No adverse events from skin inflammation or infection have been reported during the 3-year follow-up period of the BCI.

\section{Comparison with Other Studies}

Results from other similar studies are of interest, and especially comparisons with the commercially available active transcutaneous Bone Bridge (Med El). The selected comparative studies are chosen with the criteria that both tone and speech audiometry are performed in unaided and aided conditions and where at least 6 or more subjects are included [Ihler et al., 2014; Rahne and Ehelebe, 2014; Riss et al., 2014; Eberhard et al., 2016; Gerdes et al., 2016; Ihler et al., 2016; Schmerber et al., 2017]. Different methods have been applied concerning indication for the Bone Bridge, number of subjects, tone and speech audiometry, follow-up time, and the presentation of questionnaires. When comparing results from the abovementioned studies, a general conclusion is that the results are similar to this study. Both the BCI and the Bonebridge seem to improve tone thresholds and speech perception compared to the unaided situation with statistical support. There also seems to be an established benefit from the questionnaire results compared to the unaided situation. A few studies include comparison with the Bone Anchored Hearing Aid either on head band or as a percutaneous solution (different group of patients) [Gerdes et al., 2016; Ihler et al., 2016; Schmerber et al., 2017]. Also, in these aspects the comparisons with the BCI are similar. Surgery is generally without complications, and there are no reports of serious skin problems. Among the selected comparative studies, the skin in the surgical area is evaluated and described in 3 studies [Ihler et al., 2014; Eberhard et al., 2016; Schmerber et al., 2017], where there are 2 cases with postoperative wound healing resolved using only antibiotics in one case, and by both antibiotics and a minor surgical intervention in one case.

\section{Adverse Events}

After 3 years of use, the patients are satisfied with their BCI hearing aid, and no serious adverse event has occurred. Soon after the activation of the audio processor, one patient experienced a clicking sound when attaching the audio processor and also heard a scratching sound when rotating the audio processor in place, but the implant function and the sound quality were not affected when the audio processor was in place and in use. It was found that the sound phenomena were due to a loose retention magnet within the implanted titanium can. The BCI was explanted and a new device was implanted. This operation showed that the BCI could easily be removed, which is important, e.g. before a vital MRI.

\section{Conclusions}

The $\mathrm{BCI}$ has been implanted in 16 patients, and in this report, 10 patients have completed the 3-year follow-up. Warble tone thresholds, SRT, and SRS show improved results over the unaided situation at fitting and at every 
follow-up visit $1,3,6,12$, and 36 months after fitting. Compared to a Ponto Pro Power on a soft band, there was a trend of similar or better results in the majority of the parameters including SNR thresholds when using the BCI, but without statistically significant differences between the devices. Three questionnaires were completed, and the patients reported on a subjective benefit from the $\mathrm{BCI}$ intervention as well as use in different listening situations and in interaction with people around them. The results have statistical support in comparison with the unaided condition and are stable over time. Sound transmission remained unchanged, indicating good contact to the bone and consistent functionality of the whole BCI system. The force needed for using the BCI respects skin integrity without complications. For indicated patients, the $\mathrm{BCI}$ is a safe and reliable active transcutaneous BCD.

\section{Statement of Ethics}

The study was approved by Ethical Review Board (Dnr 445-12) and the Swedish Medical Products Agency (461:2012/513308). Patients gave their written informed consent for the use of their clinical records in this study.

\section{Disclosure Statement}

Ann-Charlotte Persson, Sabine Reinfeldt, Bo Håkansson, and Måns Eeg-Olofsson have been part-time consultants for Oticon Medical at some points of the clinical study. Otherwise, the authors report no conflict of interest.

\section{Funding Sources}

This study was supported by grants from the Swedish Innovations Agency (Vinnova), The Swedish Association of Hard of Hearing People (HRF), and Promobilia. This study was supported by Oticon Medical AB, Sweden.

\section{Author Contributions}

A.-C. Persson, S. Reinfeldt, B. Håkansson, K.-J. Fréden-Jansson contributed by collecting the data and writing and approving the manuscript. C. Rigato contributed by reading the manuscript and by doing the statistical analysis. M. Eeg-Olofsson contributed by supervising the study and writing and approving the manuscript. $\mathrm{He}$ was medically responsible and performed all surgical procedures.

\section{References}

Cox RM, Alexander GC. The abbreviated profile of hearing aid benefit. Ear Hear. 1995 Apr; 16(2): 176-86

Cox RM, Alexander GC. The International Outcome Inventory for Hearing Aids (IOI-HA): psychometric properties of the English version. Int J Audiol. 2002 Jan;41(1):30-5.

den Besten CA, Monksfield P, Bosman A, Skarzynski PH, Green K, Runge C, et al. Audiological and clinical outcomes of a transcutaneous bone conduction hearing implant: sixmonth results from a multicentre study. Clin Otolaryngol. 2019 Mar;44(2):144-157.

Eberhard KE, Olsen S $\varnothing$, Miyazaki H, Bille M, Caye-Thomasen P. Objective and Subjective Outcome of a New Transcutaneous Bone Conduction Hearing Device: Half-year Follow-up of the First 12 Nordic Implantations. Otol Neurotol. 2016 Mar;37(3):267-75.

Eeg-Olofsson M, Håkansson B, Reinfeldt S, Taghavi $\mathrm{H}$, Lund $\mathrm{H}$, Jansson KJ, et al. The bone conduction implant-first implantation, surgical and audiologic aspects. Otol Neurotol. 2014 Apr;35(4):679-85.

Eeg-Olofsson M, Stenfelt S, Taghavi H, Reinfeldt S, Håkansson B, Tengstrand T, et al. Transmission of bone conducted sound - correlation between hearing perception and cochlear vibration. Hear Res. 2013 Dec;306:11-20.
Fredén Jansson KJ, Håkansson B, Rigato C, EegOlofsson M, Reinfeldt S. Robustness and lifetime of the bone conduction implant - a pilot study. Med Devices (Auckl). 2019 Feb 26;12: 89-100.

Gerdes T, Salcher RB, Schwab B, Lenarz T, Maier $\mathrm{H}$. Comparison of Audiological Results Between a Transcutaneous and a Percutaneous Bone Conduction Instrument in Conductive Hearing Loss. Otol Neurotol. 2016 Jul;37(6): 685-91.

Hagerman B, Kinnefors C. Efficient adaptive methods for measuring speech reception threshold in quiet and in noise. Scand Audiol. 1995;24(1):71-7.

Håkansson B, Reinfeldt S, Eeg-Olofsson M, Ostli $\mathrm{P}$, Taghavi $\mathrm{H}$, Adler J, et al. A novel bone conduction implant (BCI): engineering aspects and pre-clinical studies. Int J Audiol. 2010 Mar;49(3):203-15.

Ihler F, Blum J, Berger MU, Weiss BG, Welz C, Canis M. The Prediction of Speech Recognition in Noise With a Semi-Implantable Bone Conduction Hearing System by External Bone Conduction Stimulation With Headband: A Prospective Study. Trends Hear. 2016 Oct;20:2331216516669330.
Ihler F, Volbers L, Blum J, Matthias C, Canis M. Preliminary functional results and quality of life after implantation of a new bone conduction hearing device in patients with conductive and mixed hearing loss. Otol Neurotol. $2014 \mathrm{Feb} ; 35(2): 211-5$.

ISO. Acoustics - Audiometric test methods. Part 3: Speech audiometry. Geneva: International Organization for Standardization; 2012. pp. 8253-3.

Jansson KJ, Håkansson B, Reinfeldt S, Rigato C, Eeg-Olofsson M. Magnetic resonance imaging investigation of the bone conduction implant - a pilot study at 1.5 Tesla. Med Devices (Auckl). 2015 Oct;8:413-23.

Kramer SE, Goverts ST, Dreschler WA, Boymans $M$, Festen JM. International Outcome Inventory for Hearing Aids (IOI-HA): results from The Netherlands. Int J Audiol. 2002 Jan;41(1): 36-41.

Rahne T, Ehelebe T. Objective estimation of frequency-specific pure-tone hearing thresholds following bone-conduction hearing aid stimulation. ScientificWorldJournal. 2014;2014: 247942.

Reinfeldt S, Håkansson B, Taghavi H, Eeg-Olofsson M. New developments in bone-conduction hearing implants: a review. Med Devices (Auckl). 2015a Jan;8:79-93. 
Reinfeldt $\mathrm{S}$, Håkansson $\mathrm{B}$, Taghavi $\mathrm{H}$, Fredén Jansson KJ, Eeg-Olofsson M. The bone conduction implant: clinical results of the first six patients. Int J Audiol. 2015b Jun;54(6):40816.

Reinfeldt S, Rigato C, Håkansson B, Fredén Jansson KJ, Eeg-Olofsson M. Nasal sound pressure as objective verification of implant in active transcutaneous bone conduction devices. Med Devices (Auckl). 2019 May;12:193-202.

Riss D, Arnoldner C, Baumgartner WD, Blineder $\mathrm{M}$, Flak S, Bachner A, et al. Indication criteria and outcomes with the Bonebridge transcutaneous bone-conduction implant. Laryngoscope. 2014 Dec;124(12):2802-6.
Robinson K, Gatehouse S, Browning GG. Measuring patient benefit from otorhinolaryngological surgery and therapy. Ann Otol Rhinol Laryngol. 1996 Jun;105(6):415-22.

Schmerber S, Deguine O, Marx M, Van de Heyning P, Sterkers O, Mosnier I, et al. Safety and effectiveness of the Bonebridge transcutaneous active direct-drive bone-conduction hearing implant at 1-year device use. Eur Arch Otorhinolaryngol. 2017 Apr;274(4): 1835-51.

Schnabl J, Wolf-Magele A, Pok SM, Schoerg P, Hirtler L, Schloegel M, et al. Intraoperative measurement for a new transcutaneous bone conduction hearing implant. Otol Neurotol. 2014 Aug;35(7):1242-7.
Shirinkar M, Ghoncheh M. Development of an intraoperative evaluation method for a novel Bone Conduction Implant using nasal sound pressure., Gothenburg, Sweden: chalmers University of Technology; 2013., Stenfelt, S., \& Reinfeldt, S. (2007). A model of the occlusion effect with bone-conducted stimulation. Int J Audiol. 2013;46(10):595-608.

Taghavi H, Håkansson B, Reinfeldt S, Eeg-Olofsson M, Jansson KJ, Håkansson E, et al. Technical design of a new bone conduction implant (BCI) system. Int J Audiol. 2015;54(10): $736-44$ 